\title{
A Novel Framework for Modelling Wheelchairs under the Realm of Internet-of-Things
}

\author{
Sameer Ahmad Bhat ${ }^{1}$, Muneer Ahmad Dar ${ }^{2}$, Hazem Elalfy ${ }^{3}$, Mohammed Abdul Matheen $^{4}$, Saadiya Shah ${ }^{5}$ \\ Dept. of Electrical and Electronics Eng., Kuwait College of Science and Technology, Doha, 93004, Kuwait ${ }^{1}$ \\ Dept. of Computer Science, National Institute of Electronics and Information Technology, Srinagar, India ${ }^{2,5}$ \\ Dept. of Computer Science and Eng., Kuwait College of Science and Technology, Doha, 93004, Kuwait ${ }^{3}$ \\ Dept. of Eng. Mathematics and Physics, Faculty of Eng., Alexandria University, Alexandria, 21544, Egypt ${ }^{3}$ \\ Dept. of Computer Science, Common First Year, King Saud University, Riyadh, Saudi Arabia ${ }^{4}$
}

\begin{abstract}
Innovations in research labs are driven to global markets by applied, established standard engineering practices, using state-of-the-art research that most likely results in manufacturing highly effective and efficient engineered products. As a technology, that enables assistance to physically challenged people, wheelchairs have attracted researchers across the globe whilst showcasing an increased demand for higher production. However, wheelchairs in relation with the environments implementing Internet-of-things (IoT) devices, have been mostly overlooked to include the assessment of global market trends. Therefore, this paper proposes Acceptability Engineering (AE) framework to enhance the growth and expansion of markets relying on the environments, wherein wheelchairs can coordinate with IoT to enable smart technologies. AE as a standard engineering approach would help in - evaluating the characteristics of IoT-wheelchair environments, analysing their market trends, and highlighting the deficiencies between early and prevailing markets. This will significantly impact the manufacturers, who market wheelchairs specific to the IoT environments, and in addition manufacturers would be able to identify the potential users of their manufactured products.
\end{abstract}

Keywords-Wheelchairs IoT; acceptability engineering; humancentered engineering; innovative technologies; early adopters

\section{INTRODUCTION}

In recent years, research has focused on analyzing various existing models of wheelchairs, so as to transform them into highly advanced innovative products to enable human assistance. Wheelchairs embed intelligent systems which enable functional control of sophisticated drive control mechanisms, and recent studies are observed to focus on improving the overall control system, drive configuration mechanism and human-machine interaction [1], [2], [3]. Besides enabling assistance to the people with locomotion needs, wheelchairs also offer support in several other application areas, wherein they operate autonomously to accomplish certain time bound critical tasks. For instance, in On-Demand Transportation in Hospital Environment [4], Open Area Path Finding [5], wheelchair users in smart city planning [6].

In today's techno-savvy world, a number of things connect to the internet to transfer or update current status information, send or receive files, and reflect changes in databases. Objects around us when connected to each other through the internet are termed as Internet of Things (IoT) [7], in which every object holds a unique identity and can be accessed from anywhere anytime, through its exposed interfaces for monitoring and control, and acquire current status information. The number of objects connecting to the internet and the object-to-object communication rate, both are growing at an extraordinary rate. Billions and trillions of context aware, selfmotivating remotely connected devices, integrate platforms for social integration and commercial business processes, for instance, social internet-of-things [8], Object with self-healing properties in IoT [7], Edge System for Smart Healthcare [9], ubiquitous computing [10], and ambient Intelligence [11], are predicted to widely expand the horizon of IoT. Presently, many IoT devices support home and business work flows, thereby ameliorate life experiences of people, as well as elevate the global market business trends. While IoT continues to dominate global markets, research in exploratory technology innovation processes specific to wheelchairs faces several challenges [12], [13], [14], and to overcome those challenges, whilst proposing concoct solutions to such problems, technology innovations in wheelchairs essentially require appropriate engineering methods.

Acceptability Engineering (AE) [15], a standard engineering approach, aims to build, estimate, and measure the effectiveness of innovative technology and users' acceptance relationships. As a systematic approach to assessment, AE reveals and overcomes the differences between early and late adopters, and between early and conventional markets. In order to determine the characteristics of technology innovation and user acceptance, AE provides a logical path for systematically analyzing such characteristics, and also helps in estimating market trends. AE primarily helps to access innovation technologies and their acceptance based on the characteristics defined by a user-centric engineering design, perception of the state-of-the-art technologies, users' realization, users' perception of information technology, and first-time user of an innovation technology.

In this paper, we aim to address the challenges that emerge as a result the two overlapping technological domains the wheelchair technology domain and IoT domain, and we attempt to provide a feasible solution covering both domains and associated challenges. The main contributions of this paper are highlighted as under:

- $\quad$ Our proposed framework provides a common systematic design and assessment framework specific to the engineering that incorporates wheelchairs and IoT.

- The proposed framework defines key assessment strategies that can be applied in the design engineering process of manufacturing wheelchairs, specifically meant to operate in IoT networks. 
- $\quad$ The proposed framework can help in accessing the role of assistive technology in relation with the IoT, as well evaluating users' perception of wheelchair under IoT domain.

- $\quad$ Our proposed framework can explain market trends to unleash the potential growth of wheelchair markets that operate under IoT networked environments, and would enable assessment of user markets, in particular users' adoption of assistive technology.

This study is organized into four different sections. The following Section II, provides a detailed background to the study. Section III describes the current trends in the context of wheelchairs. Section IV comprises a discussion on the Acceptability Engineering and IoT-Wheelchairs. Finally, Section $\mathrm{V}$ provides an overall conclusion of the study.

\section{BACKGROUND}

Currently different types of wheelchair models are found in market and are typically used in a variety of social order fields. Patients with movement disabilities, in essence, have to perform real life tasks. Most of the tasks may require a person to move on from one location to the other, and wheelchairs act as a primary motion assistance tool for the patients with locomotive disabilities. Wheelchairs fundamentally vary in hardware and software, but the major difference that categorizes them is the propelling mechanism. Wheelchairs are basically operated either by a rider whirling the wheels directly, or may inevitably require someone to push the wheelchairs from behind, however, there also exist electronic wheelchairs that are propelled by powerful electric motors for navigation and other tasks, and these are typically controlled by a microcomputer-based circuitry [16]. Patients, who are weak enough to drive wheelchairs with their muscular arm force, make use of electronic wheelchairs. These type of wheelchair designs particularly target groups of people with walking disabilities, and allow digital control of the wheelchair, which may constitute an embedded processor for directional navigation system. The navigation system is typically controlled with a joystick that acts as a main controlling device. Electric wheelchairs mounted with sensors, actuators, and embedded processing elements, are categorized as Smart wheelchairs [17]. Most of them provide some additional controls for speed, navigation, breaks, alarms, etc. and due to the rigid and metallic construction, and digitally controlled electronic subsystems, they serve as an efficient hardware resource for building autonomous/semi-autonomous navigation robots [18], step climbing wheelchairs [19], brain- controlled wheelchairs [20], gesture-controlled wheelchairs [3] and other types.

The birth of IoT has led to its application in almost every aspect of human life. Recent studies reveal rapid updates in IoT technology, which implies massive scaling and outsourcing of the IoT products in the upcoming years [21]. However, looking at wheelchairs from the perspective of IoT environments, wheelchairs can be thought of as unilateral entities, disassociated and disconnected from IoT networks, and are unexpected to work in collaborative environments. Eventually, wheelchairs do require a space in IoT environments, wherein they can share, coordinate, communicate system/user status, and location information. This motivates us to employ and merge wheelchairs, as disconnected entities, within the IoT environments, thereby leading researchers to towards the newly emerging challenges. So far, several research studies have attempted look into the scenario of wheelchair operating in association with IoT networks, and this implies more innovations are yet to come. Therefore, analyzing the development process, market growth and user acceptance of wheelchairs in the IoT based environments needs focus, and is subject to a new approach of engineering.

As IoT is an emerging field, and is still in its early phase, exploration of wheelchairs networked within IoT environments, will present imminent challenging tasks with varying complex levels. Therefore, researchers will be able to explore existing gaps in knowledge, underlying within the composite domain formed by IoT layer and the wheelchair. From our literature survey, we observe that till date there is no engineering approach that specifically defines an evaluation methodology for the wheelchairs that would operate in IoT environments. Therefore, in this study, we propose Acceptability Engineering (AE) as an engineering method to model the framework that amalgamates the domains of assistive technologies and the IoT. The AE framework can support in developing, perceiving, and evaluating the role of innovative technology as well as highlighting the users' perception of assistive technology, in particular wheelchairs. AE can uncover the gap that prevails between the novice and later users of assistive technology, abreast to enabling a systematic track of assessment of emerging trends between initial and later markets. To explain the technology innovation, and its acceptance by the end users, $\mathrm{AE}$ can help in the design engineering process of assistive technology, that operates under the umbrella of IoT, that bases on human centered approach of engineering, users' perception of innovative technology and its acceptance, users' experience of applied information technology, and the evaluation of early adopters needs of engineered assistive technology.

Therefore, the paper guides through a logical perspective of how the application of AE can assist in the evaluation of innovation processes that drive wheelchair technology in the IoT based environments, how the application of AE in the innovation process will enable researchers to evaluate the system characteristics, which include, engineering the system, identifying interdisciplinary domains, determining user skill levels, technology innovation under consideration and potential adopters of technology in the markets.

\section{CURRENT TRENDS}

The role of technology is increasing rapidly in improving mobility of physically challenged people. In order to understand the different types of human assisting technologies, specifically meant for people with disabilities, we need to setup benchmarks to compare and analyze the current technologies aimed at delivering human mobility. Using international standard assessments of World Technology Evaluation Center, the National Science Foundation began a study, which was conducted by a team of experts, in charge of collecting information related to current trends in technology, to perform mobility conversion for the people with motion disabilities [22]. Despite the limited scope of their study, the team highlighted seven mobility tasks, necessary for the development of mobility assistance. These seven tasks relate to - positioning, stability and relocation, operation, mobility, stir uphill, other motion tasks, and the transportation. While considering technology innovation for disabilities, those seven mobility tasks could serve as starting initial checklist, to assess and thoroughly define wheelchair devices. While considering technology innovation for disabilities, those seven mobility tasks could serve as starting initial checklist to assess and thoroughly define wheelchair devices. Prior research studies have attempted to 
address and unpin only some of the above-mentioned tasks, for example, intelligent wheelchair for tennis [23], development of simulator and analysis tool for navigating in indoor locations [24], adaptive motion control for semi-autonomous wheelchairs [25], hands free control for wheelchair [26] and Voice Controllable Wheelchair [27]. In the last five years, there is an elevated trend to integrate intelligence systems into wheelchair control boards [28] to enable automatic or semiautomatic wheelchairs design. The recent innovative solution proposed and developed by AT\&T for the wheelchair employ IoT, enable access to data that influence everyday lives of wheelchair users [29]. Through the remote access to this global data, stored on highly secured cloud platforms, allows data sharing among various stake holders of the system, to improve system usage and benefit the users' as well as designers.

With the Smart Technology dominating all the areas of technology nowadays, the checklist of these seven parameters is inadequate and needs extension, and this extension is further subject to the wheelchair application domains; those domains where the assistive technology is deployed for serving humans in a variety of areas other than the area of typical human mobility assistance. Some areas may necessitate inclusion and careful estimation and evaluation of all seven parameters, whereas others may need only a few. For example, if a wheelchair is employed for an individual with minor motion disability, it does not require critical monitoring, evaluation and inclusion of smart technologies in the wheelchair design. On the other hand, some wheelchair designs may require critical evaluation of above-mentioned parameters. For example, people with serious locomotive problems need time bound, frequent systematic and careful monitoring, and there is no room for failures as it may result in loss of human lives. Consequently, regular monitoring and safety measurements remain the top priorities in such systems. Most applications require mounting sensors on wheelchairs, to record each and every critical activity, happening in either real or non-real times. Real time activity monitoring in wheelchairs requires addition of the other dimensions, like network connectivity, data and information security, data storage, measurement and estimation of various hardware and software parameters, to the previous checklist of seven parameters. Monitoring and tracking wheelchair status round the clock is a difficult task as it requires someone to be always available with the wheelchair. Also, remote assistance is often preferred to control devices that are out of reach. These issues introduce us to the concept of internet of things, in which each object is viewed as a networked entity to provide activity data and information from anywhere and anytime. By analyzing these recent trends, we can briefly state the current needs, that form the core basics and are prerequisite to the design and development of wheelchairs. Following are specified trends, which serve as a guide for research scholars working towards the future research agendas in the current context.

- Modelling and development of efficient wheelchairs designs through state-of-the-art innovative technologies.

- Technology transformation and quality production, involving multi-disciplinary approach to develop highly structured innovative products.

- Understanding the recent realm of current innovative technologies and their applications.

- Devising frameworks to measure behavioral satisfaction levels of various wheelchair systems users.
- Identifying potential adopters of newly innovative technologies.

\section{ACCEPTABILITy ENGINEERING AND IoT}

To underline IoT-Wheelchair design characteristics, this study is based on frameworks primarily focusing on users' innovative technology acceptance. As discussed in the previous sections, Acceptability Engineering (AE) incarcerates the prevailing mutual relationship between innovative technology and user acceptance. It can be used to evaluate current trends and characterize wheelchair technology in relation with IoT. Fig. 1 shows the proposed framework for Wheelchair and IoT co-domain, and the following sub-sections next, describe the framework in more details.

\section{A. Perception of Human-Centered Design Engineering}

AE mainly concerns research on human-centered innovative technologies, the association between innovative technologies and user acceptance. It systematically examines users' technology acceptance behavior through cognitive, emotional and social factors. IoT-Wheelchair design more importantly concerns users' acceptance. Therefore, it is quite satisfactory to apply AE's human centric approach in the design process. The Human-centered engineering approach also called as user centered design (USD), directly places a user at the core of the design and decision-making engineering process. Human centered design approach engaged in AE defines how users accept innovative technologies, in contrast to building advance technology systems. Therefore, development of well-organized and robust products, is a natural outcome of this approach, and this will help us in - estimating factors responsible for higher user acceptance rates, designing acceptable alternatives to failing innovative technologies, evaluation of user acceptance and prediction of technology innovation success rates.

The cognitive and organizational factors are an essential construct in the product design and safety assessment processes. Accuracy level of procedure designs and interfaces can be highly improved via the human machine interaction analysis. This places responsibilities on cognitive science engineers to precisely assess the psychological behavior of IoT-wheelchair users. The primary and transient conditions observed IoT-wheelchairs, can be compared and examined through various methods and interfaces. The theories of cognition form the basis to the Human Factor Analysis Methods and are applicable to several engineering disciplines. These can be applied within four different areas of engineering: system designing, measuring safety levels, training, and accidental analysis.

Safety, is the next and important step in design process for wheelchair users, as well as the wheelchair itself. Studying IoT-wheelchair relationship, poses certain questions to the engineers. The first question to be answered is how to ensure the real time data communication. Next is to assess the system behavior based on some prior Safety Instructions Model. To support a driving wheelchair, safety driving assistance system can be setup through the IoT. As IoT offers opportunities to a number of users to synchronize and share their experiences through interconnection, it guides engineers to develop a framework for automatically adjusting the safety parameters, including context awareness. These safety parameters could be specified in the IoT model or could be employed directly in the software models of wheelchair systems. Therefore, AE can support in developing safety models based on user acceptance levels, in the context of IoT and wheelchairs. 


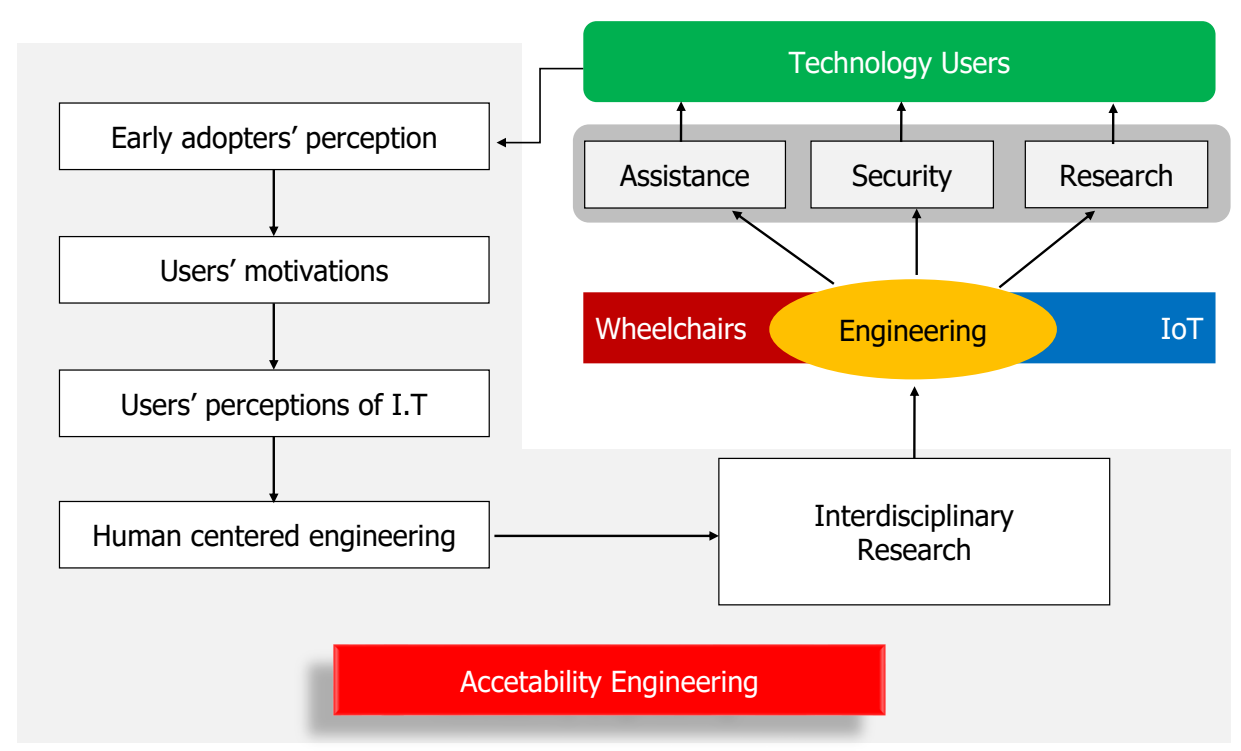

Fig. 1. Acceptability Engineering for Wheelchairs and IoT Domain.

IoT-wheelchair design and development requires it to be user-centric, with all of its components being highly natural. During our literature search, we realized that only a small number of studies have followed up with this kind of approach. Therefore, it needs to be mentioned that designing wheelchairs using human-centered design would prove to be a highly significant methodology, as its main objective is to assist and satisfy user requirements.

\section{B. Technology Perception using Interdisciplinary Research}

Wheelchair design engineers rigorously research for developing efficient hardware and software, and activities demand engineering from multiple disciplines to work collaboratively. Often, engineering teams work so closely in association, that logical domain boundaries seem overridden. This transdisciplinary approach in the design engineering process looks a singular domain with blurred boundaries between the unlike domains. To solve complex problems, the trans-disciplinary approach proves to be the best choice. Consequently, the IoTwheelchair relation requires extensive artwork of engineers to transmute wheelchair model into an IoT model. The interdisciplinary nature of AE includes systematic research methods applicable to various engineering disciplines, e.g., computer, electrical, electronics, mechanical, and industrial engineering, to devise a common framework in order to achieve a common objective. It also involves knowledge of human and social sciences, including disciplines like sociology, psychology, marketing, cognitive, and art. These disciplines are critical to $\mathrm{AE}$, and are essential for the research and analysis of people who make use of innovative technologies. Thus, applying AE in IoT-wheelchair design engineering process would result in products involving interdisciplinary domains.

\section{Perception of Innovative Technologies}

Technologies come and stay for a period of time in markets. One of the fundamental objectives of AE, is to understand user acceptance of innovative technologies, by applying the systematic and scientific exploration methods, that provide approximation and estimation of success rates of such innovations in markets. The interesting aspect here is to understand the different phases that constitute the process cycle of innovative technologies. This helps technical teams and financiers to formulate strategies to expand innovations effectively. Thus, for an innovation to be efficacious, the major concern for developers is to estimate the strength, weakness, and future perspective of such pioneering technologies. However, the theories, models, and systematic research methods to accomplish and implement such innovations, have not been entirely developed and explored yet.

The life cycle of innovative technology is the most important aspect for its assessment. Its thorough comprehension enables developers and investors to efficiently extend the scope of innovative technologies. Therefore, the processes and systematic methods for assessment of innovative technology should be taken into consideration. Developing IoT-wheelchair requires study of individual life cycles of both IoT and wheelchairs. after analyzing the underling architectures of both domains, we can then identify the different levels of both the system in order obtain to common interface levels for integration.

\section{Early Adopter's Perception of IT}

AE mainly targets information technology (IT) users, but also shifts its focus on other types of users as well. Computer technologies exist almost in every device to solve our daily tasks effectively and efficiently. For example, smart home is an intelligent/autonomous home environment setup, enables users to remotely control and manage their devices [25]. The IoT advancement has led to a wider acceptance of the concept of smart devices, and the engagement of development communities in development process is increasing rapidly, since the tools and solutions available from information communication technologies (ICTs) are scaling up every minute. Wheelchair users must have prior knowledge of sub system functions like, GPS, GSM, and other components. People with a little IT knowledge may find it difficult to operate a complex wheelchair equipped with IoT operative systems. This limits the system usage due to the lack of operating procedures. Additionally, having little knowledge may prove to be dangerous 
for both the users and to the wheelchair hardware. Nonetheless, expert users of IT can operate the device with ease, and can take full advantages of the newer system functions enabled by IoT. For example, users can be guided for safe driving, informed about status of wheelchair, GPS to show the shortest routes, GSM to send any emergency requests, Wi-Fi to provide connectivity, latest updates about traffic on routes, updates about bus services, wheelchair systems information and notifications to user and remote caregiver. With the application of AE to the IoT-wheelchairs, user acceptance perception can be evaluated by recognizing and categorizing users on the basis of their IT skill levels. The IT level of wheelchair user, plays an important role while establishing an agreement between user and the wheelchair. People, who make effective use of IT to solve simple or complex problems, have been evaluated in several studies in various disciplines like ergonomics, Human Computer Interaction, and computer-based tasks with mutual interaction among participants.

Implementation of ICT in wheelchair design urges engineers again to apply acceptability engineering, to target people who actively participate to make ICT as an important part of their lives i.e., users with unique needs would require specific ICT tools and solutions. For example, some users may expect systems to offer locomotion features based on eye movement, whereas other may require gesture recognition. Additionally, others may require some form of mapping system [26] or voice controlling [27], wire or wireless monitoring that help in navigation [23]. The IoT encompasses people who are familiar with ICTs and their usage. Therefore, the steps would be prior estimation and benchmarking the IT skill levels for users, as these skills are likely to the effective utilization of IoTwheelchairs.

\section{E. Perception of Early Adopters' Motivations}

Generally, people adopt only those technologies, which they consider as effective in improving solutions to their problems. However, different people exhibit different behavior, and pose different characteristics, so not everyone ends up in implementing the same technology for the same problem. AE aims to identify technology innovators or enthusiasts, as the key entities responsible in the innovative technology adoption process. These innovative technology enthusiasts, also called as early adopters, serve as catalysts in the technology adoption and its dissemination. The potential state-of-the-art technology adopters, whose technology acceptance decisions critically effect the adoption process, are the key sources responsible for the technology dispersion. These potential technology adopters are exclusively dependent on the technology acceptance behavior of early adopters, who direct them to either fully accept or reject newer technologies. It is difficult to predict the adoption rates of newer technologies initially, without considering the technology adoption behaviors of the early adopters. In order to fully understand and predict the recognition levels of stateof-the-art technologies, it is crucial to identify early target users, who serve as primary dispersion sources for the existing competent technologies.

In the current scenario, almost all the technological development projects, often entail in formulating some new scientific strategies and methods in the product advancement processes. This results in stimulating effects that can be directly realized in manufactured products or in the services delivered. The vital issues encountered during the planning phase, as a part of a technological implementation process, concern to users' adoption characteristics. The characteristics of Users' and technology-to-be-adopted, collectively impact the adoption behavior. Various studies have attempted to identify certain important factors responsible for assessing users' adoption behavior. Some of these factors are normative peer effect [28], self-innovativeness [29], personal characteristics, perceived technology characteristics, behavioral intentions, and managerial influence, human-related issues like time management, organizational readiness, resource limitations such as organizational users and technology key, age, full-time users vs part-time [30]. These studies contribute to measures for accessing the behavior of users towards newer technology adoption.

Typically, several studies have tried to evaluate users' technology adoption behavior by estimating three dependent variables. These are actual use, intention of use, and behavior. Engagement of user in any activity, as a result of social pressure, is termed as subjective norm. The subjective norm or social influence, which is the user's understanding of its peers' appreciation or non-recognition of the particular or universal target behavior, often succeed the above-mentioned behavioral estimation steps. Behavioral estimations, have been made in particular models only. User beliefs and social factors, are the two key elements that influence users' perception about technology acceptance and its usage. As people usually have faith in their peers or elderly people in their social network, their beliefs grow correspondingly according to the level of their peers or network connections they have. Hierarchical group dynamics and peer instigated decisions or the decisions taken by the other parties prominently impact a person's or establishments decisions in many societal and commercial situations. For example, effects could be seen in business investments, innovative technology implementations, organizations' premeditated decisions, public or private administrative voting, and custom trends.

Further, studies have proved that the social pressure by peers and elders do impact user beliefs in the technological domain. So, the users' technology acceptance rate at this juncture is dependent on the users' association with its peer or elderly network. Therefore, higher user social connections or links is directly proportionate to higher adoption rates. Observing technology adoption process through the lens of IoT-wheelchair technology, the characteristics of both the users of wheelchair and IoT-wheelchairs must be studied in relation to each other. This leads us to take up the Task-Technology Fit (TTF) model [31] as the basis for modelling the characteristics of both the users and the technology employed, which in our case are wheelchair users and IoT-wheelchairs respectively. TTF model identifies the characteristics of users and the technology, as the two important elements which ultimately lead to technology utilization. This model provides theoretical basis to know how users assess the information systems. It also tests propositions validating the background of user assessments about technologies. Theories in past studies have tried to examine the role of individuals' characteristics by using different constructs. For example, effective technology usage, experiencing herd behavior of technology stakeholders, Selfinnovativeness, Mindfulness in the face of trends.

Mirmahdi [32] has identified three highly responsible factors influencing the behavior of potential adopters. These three factors are self-efficacy, personal innovativeness, and mindfulness. The ability of a person to trust himself/herself to achieve or complete a specific task is called as self-efficacy. Users with higher level self-efficacy are passionate to explore newer technologies, as they understand it as effortless service. 
Self-efficacy helps in explaining users' attitude towards product purchase, online learning systems, decisioning systems, and smart devices. These sources indicate that self-efficacy acts as an analyst to forecast users' behavioral and usage intentions. Wheelchair users are the most visible groups seen in the community of disabled people. Due to varied physical impairments, their motivation levels are lower as compared to the motivation levels of common people. This indicates, that the wheelchair users exhibit lower self-efficacy, and hence are unable to achieve their objectives of using assistive technology, like wheelchair, with higher motivation. Accordingly, the IoT-wheelchair development needs designs that focus on delivering effortless services, targeted on incrementing users' self-efficacy levels, instead of engaging in the development of large complex systems. The users with higher self-efficacy levels, find technology usage easier and exhibit readiness in adopting newer technologies.

The second factor, personal innovativeness, is defined as readiness to try any information technology [33]. In Innovation Diffusion Theory, personal innovativeness of individuals determines the information sources they recognize in making decisions about technology adoption. Individuals in social systems, with higher innovative inspiration are less affected by the believes of other members, in relation to any technology adoption. Thus, potential adopters' personal innovativeness, or in other words cognitive characteristics, have a significant and positive impact on adoption of newer technologies. Personal innovativeness of wheelchair users, depend upon their literacy and intrinsic motivation levels. Wheelchair users, who show interest in exploiting newer technologies, depict higher technology acceptance in their lives. While novice users with lower literacy, would be unable to utilize and understand the complete features of the IoT-wheelchair. Therefore, the IoT-wheelchair design criteria must involve the evaluation of factors responsible for users' personal innovativeness, which could also lead to higher acceptance of risk by users' employing latest technologies.

Lastly, the third factor, Mindfulness, is a state of cognizance that consist of lively information processing, and formulation and improvement of dissimilarities, while identifying various varying perceptions. Individuals with mindful abilities are able to adapt to open environments due to their tendency towards consciousness about potential technological shifts. This encourages them to manage and handle uncertainties with lower anxiety and stress levels [33]. The stress and anxiety levels of disabled people could vary from one individual to the other. Individuals with minor disability reflect least distress patterns, whereas others with major disability problems are prone to higher cognitive load. Therefore, IoT-wheelchair users may show different behavior depending upon their cognitive load. Users with mindfulness capabilities may adopt technologies quickly, whereas other users may take longer times to adopt, due to their herd behavioral characteristics, as mentioned previously.

Observations on technology characteristics, made by the decision makers, reveal that technology characteristics significantly impact the adoption behavior of potential adopters. Technologies are accepted by users only, if it satisfies users' requirements. IoT-wheelchair designing presently requires extensive research and development to enhance current disability assistance features. Technology characteristics adequately effect on TAM relationships. In order to understand and analyze technology characteristics, this requires theoretical support to clearly explain the characteristics of technology under con- sideration in relation to the technology adoption. Innovation Diffusion Theory (IDT) analyzes technology characteristics in association with technology adoption process. Rogers's, IDT explains how user's observation of technology specific information stimulates its technology adoption/rejection decision. To define technology characteristics, Rogers [33] proposes five key attributes (see Table I):

TABLE I. ATTRIBUtES OF TECHNOLOGY CHARACTERIZATION [33]

\begin{tabular}{|ll|}
\hline Technology Improvement level & $\begin{array}{l}\text { Degree of perception of a new technol- } \\
\text { ogy, as being better than its antecedent. }\end{array}$ \\
\hline Compatibility level & $\begin{array}{l}\text { Degree of perception of a new tech- } \\
\text { nology, as being reliable with adopters' } \\
\text { requirements. }\end{array}$ \\
\hline Complexity level & $\begin{array}{l}\text { Degree of perception of a new technol- } \\
\text { ogy, as being challenging to use. }\end{array}$ \\
\hline User recognition level & $\begin{array}{l}\text { Degree of results of a technology, recog- } \\
\text { nizable to others. }\end{array}$ \\
\hline Experimentation and flexibility level & $\begin{array}{l}\text { Degree of experimentation in a technol- } \\
\text { ogy, before its adoption. }\end{array}$ \\
\hline
\end{tabular}

However, some researchers [34], [35] argue that only few characteristics are essential in the process of explaining and understanding the innovation technology adoption. Out of the five key attributes, the most effective ones are - relative advantage, complexity, and compatibility frequently impact adoption. Relative advantage is one of the most commonly verified characteristics and consistent analyzer of adoption behavior. A study by Moore and Benbasat [36] reveals that technological devices' relative advantage is completely related with the adoption rate. IoT and wheelchair technology relationship can thus be studied using relative advantage, which would depict the adoption behavior. Compatibility of IoT with wheelchair technology is a factor that will be a strong driver for wheelchair technology adoption, as compatibility intensely drives technology acceptance. Similarly, users' compatibility with wheelchair technology will be the other factor accountable for the increased adoption rates. Incompatibility in the dimensions of IoT-wheelchairs may lead potential technology acceptors to recognize higher insecurity in adoption and heavily shift their focus on the impacts of identity and count of potential adopters. The complexity factor in IoT-wheelchair is critical determinant of its adoption. Complex innovations demand higher learning costs for their adoption. Thus, the IoTwheelchair complexity must be reduced to minimum levels in order to satisfy user requirements to a maximum extent. If an IoT-wheelchair system is perceived as complex by its users, then it will result in reduced attitude and motivation levels toward adoption of such newer technologies. Therefore, potential adopters' perception of technology will depend upon perceived complexity factors as is evident from the studies.

\section{CONCLUSION}

In this study, we proposed Acceptability Engineering framework as a standard approach to access the design perspective of engineering assistive technology, in particular wheelchair under the realm of IoT. The study highlights certain essential aspects, necessary for the design and development of wheelchairs in relation to the IoT. Using $\mathrm{AE}$ to extend wheelchairs with IoT, seems to be the most appropriate method for solving the issues emerging in the intercorrelation of IoT and wheelchair technology domain. The proposed model can be evaluated experimentally. Factors responsible for the increased production and market growth can be estimated. We presume industries manufacturing wheelchairs can receive potential benefits, whilst their production basis can be guided through the proposed framework. Moreover, further research is 
prerequisite to enable integration of wheelchairs in the domain of IoT. Therefore, we anticipate that our proposed framework will enable research scholars, who are actively engaged in the design of assistive technologies, to widen the scope of this study from each of the highlighted perspectives.

\section{REFERENCES}

[1] R. Cruz, V. Souza, T. B. Filho, and V. Lucena, "Electric powered wheelchair command by information fusion from eye tracking and bci," in 2019 IEEE International Conference on Consumer Electronics (ICCE), 2019, pp. 1-2.

[2] D. P. Salgado, R. Flynn, E. L. M. Naves, and N. Murray, "The impact of jerk on quality of experience and cybersickness in an immersive wheelchair application," in 2020 Twelfth International Conference on Quality of Multimedia Experience (QoMEX), 2020, pp. 1-6.

[3] M. Mahmood, M. F. Rizwan, M. Sultana, M. Habib, and M. H. Imam, "Design of a low-cost hand gesture controlled automated wheelchair," in 2020 IEEE Region 10 Symposium (TENSYMP), 2020, pp. 1379-1382.

[4] A. Baltazar, M. R. Petry, M. F. Silva, and A. P. Moreira, "Driverless wheelchair for patient's on-demand transportation in hospital environment*," in 2020 IEEE International Conference on Autonomous Robot Systems and Competitions (ICARSC), 2020, pp. 158-163.

[5] A. Basiri, "Open area path finding to improve wheelchair navigation," arXiv preprint arXiv:2011.03850, 2020.

[6] T. Götzelmann and J. Kreimeier, "Towards the inclusion of wheelchair users in smart city planning through virtual reality simulation," in Proceedings of the 13th ACM International Conference on PErvasive Technologies Related to Assistive Environments, 2020, pp. 1-7.

[7] J. P. Dias, B. Lima, J. P. Faria, A. Restivo, and H. S. Ferreira, "Visual self-healing modelling for reliable internet-of-things systems," in International Conference on Computational Science. Springer, 2020, pp. $357-370$.

[8] M. A. Azad, S. Bag, F. Hao, and A. Shalaginov, "Decentralized selfenforcing trust management system for social internet of things," IEEE Internet of Things Journal, vol. 7, no. 4, pp. 2690-2703, 2020.

[9] P. P. Ray, D. Dash, and D. De, "Intelligent internet of things enabled edge system for smart healthcare," National Academy Science Letters, pp. 1-6, 2020.

[10] J. E. Bardram and A. Matic, "A decade of ubiquitous computing research in mental health," IEEE Pervasive Computing, vol. 19, no. 1, pp. $62-72,2020$.

[11] A. Haque, A. Milstein, and L. Fei-Fei, "Illuminating the dark spaces of healthcare with ambient intelligence," Nature, vol. 585, no. 7824, pp. 193-202, 2020.

[12] D. Z. Morgado-Ramirez, G. Barbareschi, M. Kate Donovan-Hall, M. Sobuh, N. Elayyan, B. T. Nakandi, R. Tamale Ssekitoleko, j. Olenja, G. Nyachomba Magomere, S. Daymond et al., "Disability design and innovation in computing research in low resource settings," in The 22nd International ACM SIGACCESS Conference on Computers and Accessibility, 2020, pp. 1-7.

[13] A. Alshangiti, M. Alhudaithi, and A. Alghamdi, "Human factors in the design of wheelchair tray tables: User research in the co-design process," in International Conference on Human-Computer Interaction. Springer, 2020, pp. 18-24.

[14] R. J. Gowran, A. Clifford, A. Gallagher, J. McKee, B. O'Regan, and E. A. McKay, "Wheelchair and seating assistive technology provision: a gateway to freedom," Disability and Rehabilitation, pp. 1-12, 2020.

[15] H.-C. Kim, "Acceptability engineering: the study of user acceptance of innovative technologies," Journal of applied research and technology, vol. 13, no. 2, pp. 230-237, 2015.

[16] S. A. Parthasarathy, S. Subash, A. Devaraj, and V. Karthik, "Design and development of arduino based multipurpose wheelchair for disabled patients," in 2020 Third International Conference on Smart Systems and Inventive Technology (ICSSIT), 2020, pp. 884-888.

[17] Q. Liu, W.-S. Zhao, and Y. Yu, "Rfid-based bidirectional wireless rollover sensor for intelligent wheelchair," Microwave and Optical Technology Letters, 2020.
[18] C. Wang, M. Xia, and M. Q.-H. Meng, "Stable autonomous robotic wheelchair navigation in the environment with slope way," IEEE Transactions on Vehicular Technology, vol. 69, no. 10, pp. 1075910771, 2020.

[19] S. R. Thamel, R. Munasinghe, and T. Lalitharatne, "Motion planning of novel stair-climbing wheelchair for elderly and disabled people," in 2020 Moratuwa Engineering Research Conference (MERCon). IEEE, 2020, pp. 590-595.

[20] H. Li, L. Bi, and J. Yi, "Sliding-mode nonlinear predictive control of brain-controlled mobile robots," IEEE Transactions on Cybernetics, 2020.

[21] N.-N. Dao, T.-T. Nguyen, M.-Q. Luong, T. Nguyen-Thanh, W. Na, and S. Cho, "Self-calibrated edge computation for unmodeled time-sensitive iot offloading traffic," IEEE Access, vol. 8, pp. 110316-110323, 2020.

[22] R. E. Cowan, B. J. Fregly, M. L. Boninger, L. Chan, M. M. Rodgers, and D. J. Reinkensmeyer, "Recent trends in assistive technology for mobility," Journal of neuroengineering and rehabilitation, vol. 9, no. 1, pp. 1-8, 2012.

[23] K. Matsuo and L. Barolli, "Prediction of rssi by scikit-learn for improving position detecting system of omnidirectional wheelchair tennis," in International Conference on Broadband and Wireless Computing, Communication and Applications. Springer, 2019, pp. 721-732.

[24] G. Vailland, Y. Gaffary, L. Devigne, V. Gouranton, B. Arnaldi, and M. Babel, "Vestibular feedback on a virtual reality wheelchair driving simulator: A pilot study," in Proceedings of the 2020 ACM/IEEE International Conference on Human-Robot Interaction, 2020, pp. 171179.

[25] M. Ali, A. A. Ali, A.-E. Taha, I. B. Dhaou, and T. N. Gia, "Intelligent autonomous elderly patient home monitoring system," in ICC 20192019 IEEE International Conference on Communications (ICC). IEEE, 2019, pp. 1-6.

[26] M. J. Haddad and D. A. Sanders, "Deep learning architecture to assist with steering a powered wheelchair," IEEE Transactions on Neural Systems and Rehabilitation Engineering, 2020.

[27] P. P. Dutta, A. Kumar, A. Singh, K. Saha, B. Hazarika, A. Narzary, and T. Sharma, "Design and development of voice controllable wheelchair," in 2020 8th International Conference on Reliability, Infocom Technologies and Optimization (Trends and Future Directions)(ICRITO). IEEE, 2020, pp. 1004-1008.

[28] M. Conner, "Theory of planned behavior," Handbook of Sport Psychology, p. 3, 2020.

[29] Y. K. Dwivedi, N. P. Rana, K. Tamilmani, and R. Raman, "A metaanalysis based modified unified theory of acceptance and use of technology (meta-utaut): A review of emerging literature," Current Opinion in Psychology, 2020.

[30] S. Aljarboa and S. J. Miah, "Assessing the acceptance of clinical decision support tools using an integrated technology acceptance model," arXiv preprint arXiv:2011.14315, 2020.

[31] V. Z. Vanduhe, M. Nat, and H. F. Hasan, "Continuance intentions to use gamification for training in higher education: Integrating the technology acceptance model (tam), social motivation, and task technology fit (ttf)," IEEE Access, vol. 8, pp. 21 473-21 484, 2020.

[32] M. Darban and H. Amirkhiz, "Herd behavior in technology adoption: The role of adopter and adopted characteristics," in 2015 48th Hawaii International Conference on System Sciences. IEEE, 2015, pp. 35913600

[33] J. A. García-Avilés, "Diffusion of innovation," The International Encyclopedia of Media Psychology, pp. 1-8, 2020.

[34] L. G. Tornatzky and K. J. Klein, "Innovation characteristics and innovation adoption-implementation: A meta-analysis of findings," IEEE Transactions on engineering management, no. 1, pp. 28-45, 1982.

[35] J. W. Arts, R. T. Frambach, and T. H. Bijmolt, "Generalizations on consumer innovation adoption: A meta-analysis on drivers of intention and behavior," International Journal of Research in Marketing, vol. 28, no. 2, pp. 134-144, 2011.

[36] G. C. Moore and I. Benbasat, "Development of an instrument to measure the perceptions of adopting an information technology innovation," Information systems research, vol. 2, no. 3, pp. 192-222, 1991. 